Review article

Paediatrics Today 2016;12(1):49-55

DOI $10.5457 / \mathrm{p} 2005-114.135$

\title{
CONTRAST-ENHANCED ULTRASONOGRAPHY IN CHILDREN
}

\section{Damjana KLJUČEVŠEK*}

University Clinical Centre, Children's Hospital Ljubljana, Ljubljana, Slovenia

\author{
${ }^{*}$ Corresponding author: \\ damjana.kljucevsek@gmail.com \\ Tel.: + 38615229264 \\ Fax.: + 38615222993
}

Received: November 1, 2015

Accepted: November 29, 2015

Key words: Contrast-enhanced ultrasonography (CEUS) • Voiding urosonography - Safety - Children Indication.

\begin{abstract}
Development of ultrasound contrast agents (UCAs) opened the possibility of a new radiation-free diagnostic technique called contrast-enhanced ultrasonography (CEUS) in pediatric imaging. First, the safety profile of CEUS is discussed, followed by the description of common clinical applications. UCAs are not registered for individuals younger than 18 years and their current use is off-label. The published studies concerning UCA safety have shown the high safety profile of UCAs. Following intravenous application of UCAs, only one severe anaphylactic reaction has been reported in children, along with some mild adverse reactions, while during intravesical application no adverse effect related to UCAs has been reported. The main indication in the pediatric population for UCA application is voiding urosonography (VUS). The generally accepted standard VUS procedure is described together with its role in the urodiagnostic algorithm. The intravenous application of UCA is less widespread, but it is slowly gaining popularity among pediatric radiologists as a problem solving method. Unfortunately, there has been no standardization of the procedure and there are no official recommendations regarding the dose of UCAs, which should be appropriately adjusted according to the patient's weight or age, and to the probe. The review of the published literature concerning intravenous CEUS is presented. A step forward towards functional imaging is dynamic CEUS, which enables quantification of enhancement. In addition, the role of the EFSUMB Paediatric Registry Data Base is presented. Conclusion - CEUS examinations have opened new prospects in ultrasound and have become promising non-invasive radiation-free method in children,
\end{abstract}

\section{Introduction}

Contrast-enhanced ultrasonography (CEUS) in children is becoming an important modality in pediatric radiology. Ultrasound contrast agents (UCAs) in general have not been registered for individuals younger than 18 years of age. Off-label use of UCA in children makes the application of the CEUS questionable because of legal issues. On the other hand, there is a need for diagnostic innovation and child-friendly imaging in daily clinical routine (1-5). CEUS has a number of distinct advantages over computer tomography $(\mathrm{CT})$ and magnetic resonance (MRI). It can be performed immediately following ultrasound (US) examination in a variety of settings (bedside, operating theatre), operates in real time (rapid changes can be recorded and evaluated), and it is a radiation-free and inexpensive method. Second-generation UCAs are eliminated through the lungs. Therefore, poor kidney function is not a contraindication for UCA application. Factors contrib- 
uting to the growth of pediatric CEUS described by Darge et al. include: the availability of stabilized commercial UCA, development of advanced US imaging equipment for use with UCA, greater ease of performing US on a child's smaller body and more favorable tissue composition as compared to an adult, the relatively low cost and widespread availability of US equipment, and growing public concern about increasing utilization of radiation-based diagnostic imaging, especially in children (5).

Some independent professional societies have published guidelines and clinical recommendations for the safe and appropriate use of CEUS in children. They are based on comprehensive literature surveys, including results from prospective clinical trials, and incorporate many indications, despite it being currently off-label. The European Federation of Societies of Ultrasound in Medicine and Biology (EFSUMB) first incorporated pediatric CEUS in its 2008 updated clinical practice guidelines and recommendations for CEUS, and updated them in $2011(6,7)$. A "Pediatric CEUS Data Registry" has been established on the EFSUMB website, which allows data input in a prospective manner and records any adverse events. In addition, the European Society of Paediatric Radiology (ESPR) Uroradiology Task Force and the European Society of Urogenital Radiology (ESUR) Paediatric Working Group published recommendations for pediatric CEUS imaging, primarily for intravesical administration, in 2008, 2012 and 2014 (8-10). There is a great difference between official statements on CEUS in Europe and Asia on the one hand and the United States on the other. In the United States intravenous and intravesical CEUS examinations are not integrated into clinical practice.

The safety profile of UCAs and their current clinical applications are described and discussed below.

\section{Safety issues of ultrasound contrast agents}

In pediatrics, many drugs have not been tested by randomized trials and thus are not specifically licensed for use in children. The same is true for UCAs. Unfortunately, rules, which are designed to protect patients, occasionally limit the potential benefits for patients. Off-label use is a common and legal problem. Probably the most important aspect of the use of second-generation UCAs in pediatrics is their pharmacological safety. The rate of adverse effects connected with the use of UCAs is lower compared to other contrast agents in the adult population (11). The safety of UCAs, either intravenous or intravesical, has been evaluated in several studies, also in the pediatric populations. Recently a meta-analysis of adverse effects after intravenous application of second-generation UCAs was performed by Piskunowicz et al. (12). Only one severe anaphylactoid reaction in a child following the intravenous administration of UCA was described. In some children some minor adverse reactions (urtica and rash, a brief alteration of taste sensation, mild tinnitus, light-headedness) were recorded. The European survey and meta-analysis of Darge et al. evaluated the intravesical use of UCA in more than 7000 children $(13,5)$. Only $0.8 \%$ transient adverse events were described, such as dysuria, hematuria, urinary retention, urinary tract infection, perianal irritation, abdominal or urethral discomfort etc. These are more likely related to bladder catheterization than the UCA (5). All these studies showed the high safety profile of UCA administered either intravesically or intravenously. However, the smallest possibility of adverse reactions requires the undertaking of appropriate precautionary measures.

The importance of bioeffects related to UCA in the clinical setting is uncertain. It is known that the bioeffects of contrastaided diagnostic ultrasound take place on a 
microscopic scale. UCAs are suspensions of gas bodies (stabilized microbubbles). The interaction of ultrasound pulses with these gas bodies causes their destabilization and acoustic cavitation. They may act as inertial cavitation nuclei. Bioeffects on nearby cells or tissues are caused by fluid jets, extreme heating, and free radicals generated when the microbubbles collapse (14). The American Institute for Ultrasound in Medicine stated that imaging with UCA should be performed under MI below 0.4, with a minimal agent dose, and short examination time (15).

It is of major importance to obtain written informed consent before the examination, after a detailed explanation of the examination, procedure and clinical value, and the safety of UCA.

\section{Contrast-enhanced voiding cysto-urethro sonography}

Contrast-enhanced voiding urosonography (VUS) using intravesically administered
UCA is a pediatric specific application for vesicoureteric reflux detection and grading, and for urethral imaging using a transperineal approach. The VUS procedure and grading are well standardized. The basic procedural steps of echo-enhanced VUS encompass: baseline pre-contrast US of the kidneys and bladder, the bladder catheterization and intravesical administration of normal saline and UCA, and repeated scans of the bladder and kidneys during bladder filling and voiding (Fig. 1). The post-contrast scan includes uretheral imaging during voiding. Contrastspecific software dedicated to second-generation USCA should be used during the procedure. The MI is set in the low specific mode (below 0.10). The second-generation UCA is used in small doses (16). The grading of VUR is similar to X-ray voiding cystourethrography - five grades (17). Some authors used the three-grade system, similar to radionuclide voiding cystography, or the upgraded three-grade system (18).

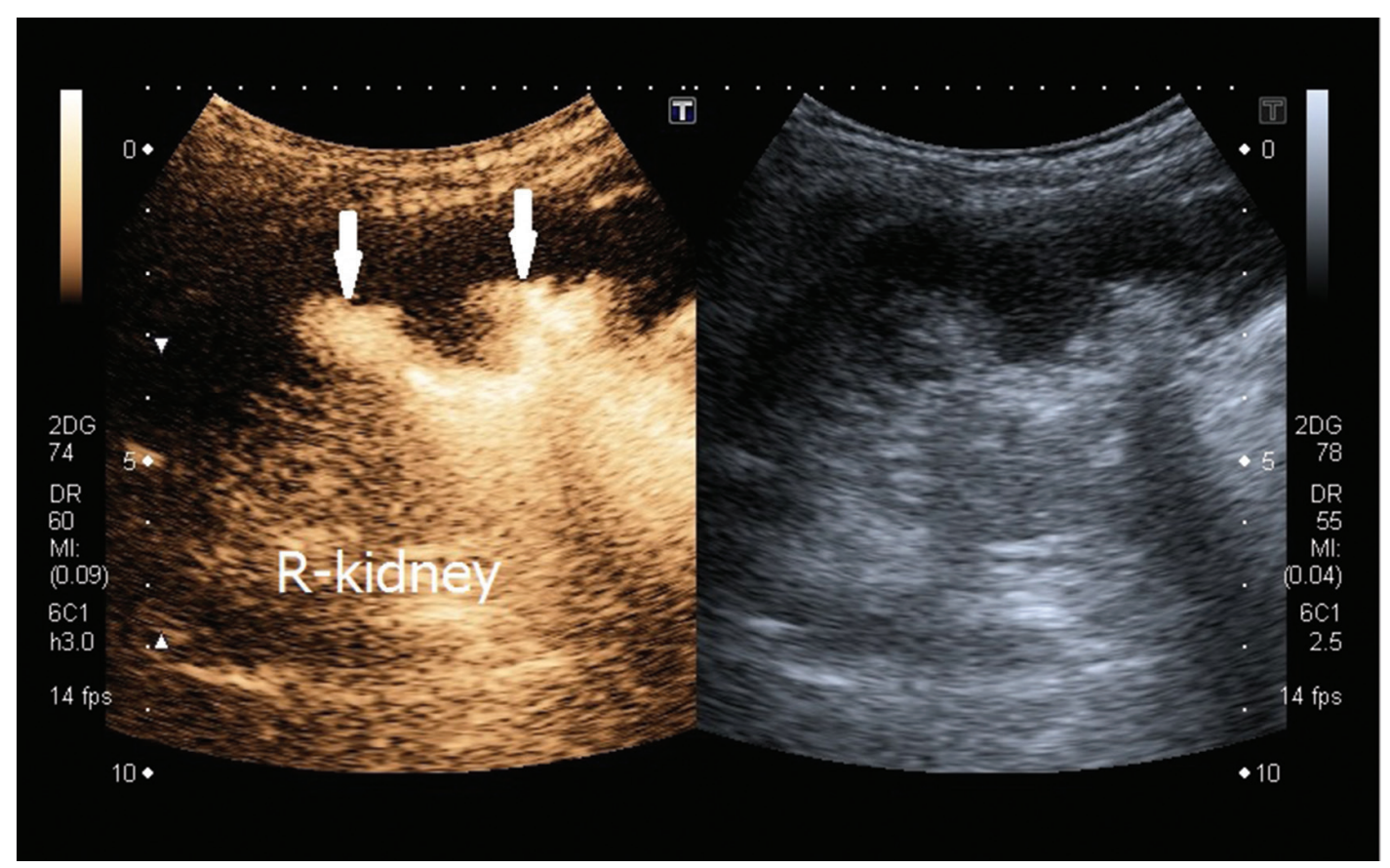

Fig. 1 Contrast-enhanced voiding ultrasonography (VUS): ultrasound contrast agent is seen in pelvicalyceal system (white arrows) in right kidney. Vesicoureteric reflux grade III is confirmed. 
The diagnostic accuracy of VUS, in terms of VUR detection and grading, has been well-established and documented. It has been evaluated in a number of comparative studies with VCUG and RNVC. All these studies were collected and analyzed in two extensive reviews, which have shown that more VUR were detected by echo-enhanced VUS compared to VCUG. In addition, the refluxes missed by VCUG were of higher grades compared to those missed by VUS, which might be important in the management of children (19). These results suggested echo-enhanced VUS as a method of choice when looking for VUR. It was proved to be a safe, highly sensitive, and radiation-free alternative for the detection and grading of VUR in children.

VCUS and RNVC are increasingly being replaced by VUS. The selection criteria for VUS are becoming wider in many medical centers and include not only follow-up examinations, first examinations in girls and screening high-risk patients, but also in children younger than one, including boys without voiding problems. There are recently more reports of imaging the urethra by VUS, which showed that imaging of the urethra is no longer a limitation of the techniques (20). Therefore, VUS is well accepted in the pediatric community in Europe and has been integrated in everyday clinical routine through a urodiagnostic algorithm according to the ESPR Uroradiology Task Force (10).

\section{Intravenous contrast-enhanced ultrasonography}

Intravenous application of UCA is less common in children and thus performed only in some medical centers. Most of the published articles report the use of CEUS as a case report for different diagnostic challenges (from spleen trauma evaluation to characterization of different parenchymal lesions in the abdomen, and contrast enhanced echocardiology).
Only a few original research papers have been published evaluating the role of intravenous CEUS: evaluation of liver and spleen injuries after blunt abdominal trauma (21), postoperative evaluation of complications after liver transplantation (22), evaluation of liver lesions and portal vein anomalies $(23,24)$, and evaluation of solid tumors in the abdomen $(25,26)$ (Fig. 2).

Piskunowicz et al. performed a meta-analysis of published papers related to intravenous CEUS in children in 2012 (27), while Schreiber-Dietrich et al. provide a summary of the available literature describing the use of intravesical and intravenous CEUS in children in 2015 (2).

In addition, dynamic contrast-enhanced ultrasound (DCE-US) enables objective evaluation of the enhancement. Quantification of DCE-US has several advantages, besides the objective evaluation of data. DCE-US enables the comparison of imaging techniques, evaluation of new UCA applications, quantification of tissue and tumor enhancement in order to characterize focal lesions, and evaluation of therapeutic response. It also limits the variability in clinical diagnosis (28). Tumor vascularity evaluation is the main clinical indication of DCE-US, but it can also be used for therapy evaluation of inflammatory bowel disease (29). When performing DCE-US the equipment settings, patient preparation, data recording and choice of one of the available quantification software packages have a great influence on the interpretation of the timeintensity curve obtained for quantification.

There are many unsolved problems, besides off-label use, for easier implementation of intravenous CEUS. First is the lack of procedure standardization. Second, there is no approved official written recommendation of the dose of UCA for different intravenous applications related to the child's weight. According to Riccabona et al. the maximum single dose for intravenous application from the neonatal 


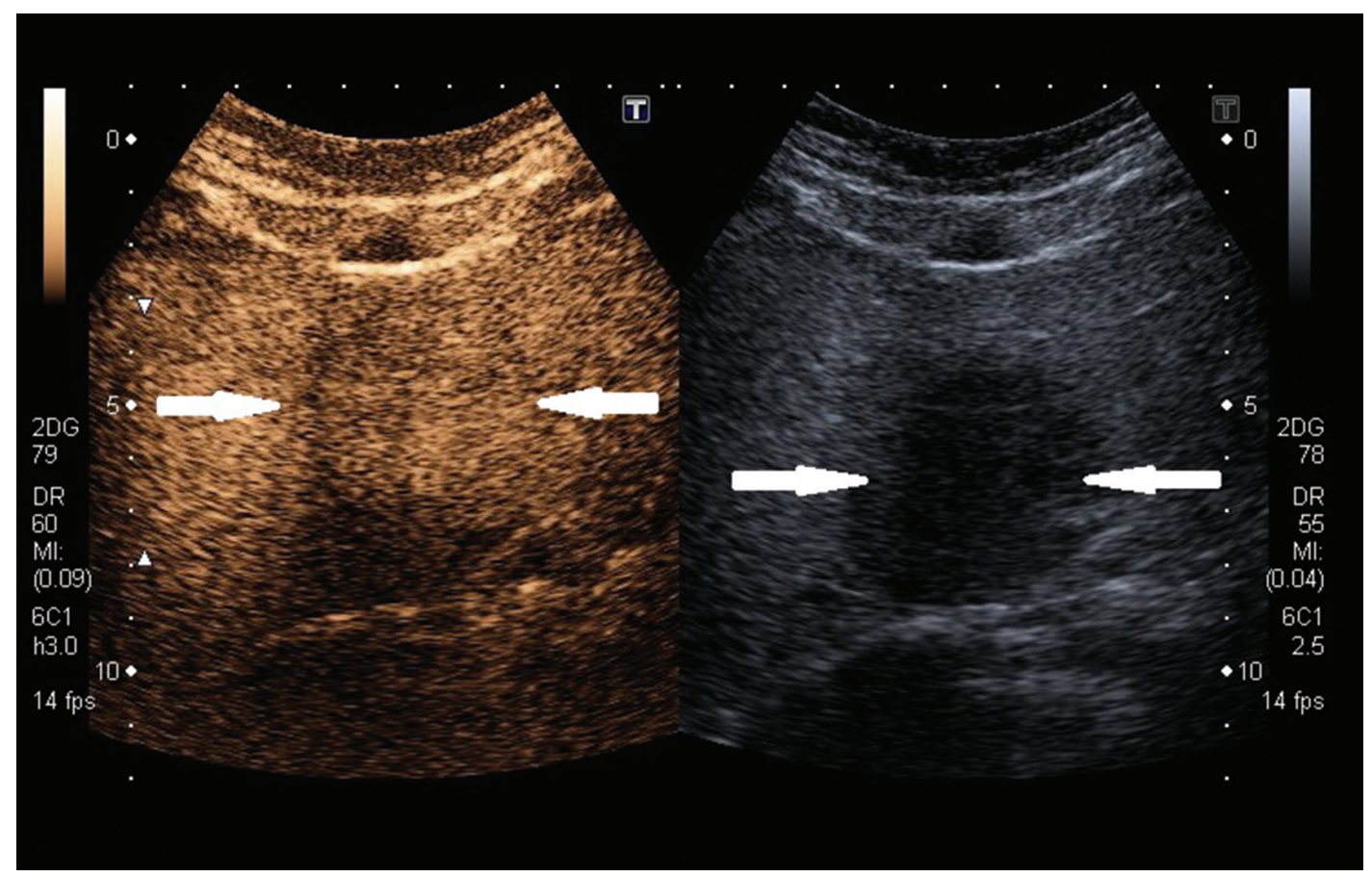

Fig. 2 Contrast-enhanced ultrasonography (CEUS) of liver as problem solving method: hypoechoic lesion in fatty liver (white arrows) seen on ultrasonography was confirmed to be focally spared area of fatty liver infiltration. During the CEUS uniform enhancement of lesion similar to normal liver parenchyma was observed. No further diagnostic imaging is necessary.

period to 3 years of age is $0.07-0.1 \mathrm{ml} / \mathrm{kg}$ body weight, from 3 to 15 years of age $0.06 \mathrm{ml} / \mathrm{kg}$ body weight or a volume $(\mathrm{ml})$ equivalent to age (years) divided by 20 (10). The dose should not be adapted only to the patient's weight or age, but also to the probe (convex, linear).

EFSUMB has created a database (EFSUMB Scientific Corner) to collect European experiences in the use of CEUS in children. The aim is to demonstrate the safety, efficacy and efficiency of this method and encourage radiologists to perform particularly intravenous CEUS and contribute the data to this database. In this way everybody is able to support the efforts of the EFSUMB to obtain approval for UCA use in pediatric patients.

\section{Conclusion}

CEUS, particularly its intravesical application (VUS), is recognized as a safe and effec- tive alternative imaging option for children in Europe. Intravenous CEUS in children is not so widespread, but initial studies in children and the wide experience in adults are promising. Although not approved for use in children, there are numerous publications supporting its safety, its clinical importance, and its value as a problem solving method in childhood. It is recommended that radiologists submit their experiences with CEUS in children to the EFSUMB Pediatric Registry and thus support the idea of open access to CEUS in pediatrics and its benefits.

Conflict of interest: The author declares that she has no conflict of interest.

\section{References}

1. Sellars ME, Deganello A, Sidhu PS. Paediatric contrast-enhanced ultrasound (CEUS): a technique that requires co-operation for rapid imple- 
mentation into clinical practice. Ultraschall Med. 2014;35:203-6.

2. Schreiber-Dietrich D, Cui XW, Piscaglia F, Gilja $\mathrm{OH}$, Dietrich CF. Contrast Enhanced Ultrasound in Pediatric Patients: A Real Challenge. Z Gastroenterol. 2014;52:1178-84.

3. Piskunowicz M, Kosiak W, Irga N. Primum non nocere? Why can't we use second generation ultrasound contrast agents for the examination of children? Ultraschall Med. 2011;32(1):83-6.

4. Dietrich D, Dietrich CF. Contrast enhanced ultrasound (CEUS) and off-label use (in children). Ultraschall Med. 2012;33(3):295-6.

5. Darge K, Papadopoulou F, Ntoulia A, Bulas DI, Coley BD, Fordham LA et al. Safety of contrastenhanced ultrasound in children for non-cardiac applications. Pediatr Radiol. 2013;43:1063-73.

6. Claudon M, Cosgrove D, Albrecht T, Bolondi L, Bosio M, Calliada F, et al. Guidelines and good clinical practice recommendations for contrast enhanced ultrasound (CEUS) - update 2008. Ultraschall Med. 2008;29(1):28-44.

7. Piscaglia F, Nolsøe C, Dietrich CF, Cosgrove DO Gilja $\mathrm{OH}$, Bachmann Nielsen $\mathrm{M}$, et al. The EFSUMB guidelines and recommendations on the clinical practice of contrast-enhanced ultrasound (CEUS): update 2011 on non-hepatic applications. Ultraschall Med. 2012;33:33-59.

8. Riccabona M, Avni FE, Blickman JG et al. Imaging recommendations in pediatric uroradiology: minutes of the ESPR workgroup session on urinary tract infection, fetal hydronephrosis, urinary tract ultrasonography and voiding cystourethrography. Pediatr Radiol. 2008;38:138-44.

9. Riccabona M, Avni FE, Damasio MB, OrdingMüller LS, Blickman JG, Darge K, et al. ESPR Uroradiology taskforce and ESUR Paediatric Working Group - Imaging recommendations in paediatric uroradiology, part V: childhood cystic kidney disease, childhood renal transplantation and contrast-enhanced ultrasonography in children. Pediatr Radiol. 2012;42:1275-83.

10. Riccabona M, Vivier PH, Ntoulia A, Darge K, Avni F, Papadopoulou F, Damasio B, Ording-Muller LS, Blickman J, Lobo ML, Willi U, et al. ESPR uroradiology task force imaging recommendations in paediatric uroradiology, part VII: standardised terminology, impact of existing recommendations, and update on contrast-enhanced ultrasound of the paediatric urogenital tract. Pediatr Radiol. 2014;44:1478-84.
11. Piscaglia F, Bolodini L. The safety of SonoVue in abdominal applications. Retrospective analysis of 23188 investigations. Ultrasound Med Biol. 2006;32:1369-75.

12. Piskunowicz M, Kosiak W, Batko T, Piankowski A, Połczyńska K, Adamkiewicz-Drożyńska E. Safety of intravenous application of second-generation ultrasound contrast agent in children: prospective analysis. Ultrasound Med Biol. 2015;41(4):10959.

13. Riccabona M. Application of a second-generation US contrast agent in infants and children: A European questionnaire-based survey. Pediatr Radiol. 2012;42:1471-80.

14. Miller DL, Averkiou MA, Brayman AA, Everbach CE, Holland CK, Wible JH Jr, et al. Bioeffects Considerations for Diagnostic Ultrasound Contrast Agents. J Ultrasound Med. 2008;27:611-32.

15. American Institute of Ultrasound in Medicine (AIUM) Statement. Bio-effects of diagnostic ultrasound with gasbody contrast agents; 2008 [updated 2015 March 25; cited 2015 Nov1]. Available from: http://www.aium.org/officialStatements/25.

16. Darge K. Voiding urosonography with US contrast agent for diagnosis of vesicoureteric reflux in children: an update. Pediatr Radiol. 2010;40:956-62.

17. Darge K, Troeger J. Vesicoureteral reflux grading in contrast-enhanced voiding urosonography. Eur J Radiol. 2002;43:122-8.

18. Kersnik Levart T, Ključevšek D, Kenda RB. How to Detect Vesicoureteric Reflux in Children? J Clin Nephrol Res. 2015;2(1):1016.

19. Darge K, Ntoulia K, Evangelou V, Papadopoulou F. A meta-analysis of the diagnostic performance of contrast enhanced voiding urosonography (ceVUS). Pediatr Radiol. 2012;42(Suppl 2):S259-S260.

20. Duran Feliubadaló C, Soler LR, Martinez CM, Novell Teixido F. Voiding urosonography: normal and abnormal appearance of the urethra. Pediatr Radiol. 2011;41(Suppl 1):S280-1.

21. Piskunowicz M, Kosiak W, Batko T. Intravenous Application of Second-Generation Ultrasound Contrast Agents in Children: A Review of the literature. Ultraschall in Med. 2012;33:135-40.

22. Valentino M, Serra C, Pavlica P, Labate AM, Lima $\mathrm{M}$, Baroncini S, et al. Blunt abdominal trauma: diagnostic performance of contrast-enhanced US in children - initial experience. Radiology. 2008;246:903-9. 
23. Bonini G, Pezzotta G, Morzenti C, Agazzi R, Nani R. Contrast-enhanced ultrasound with SonoVue in the evaluation of postoperative complications in pediatric liver transplant recipients. J Ultrasound. 2007;10(2):99-106.

24. Jacob J, Deganello A, Sellars ME, Hadzic N, Sidhu PS. Contrast enhanced ultrasound (CEUS) characterization of grey-scale sonographic indeterminate focal liver lesions in pediatric practice. Ultraschall in Med. 2013;34:529-40.

25. Pschierer K, Grothues D, Rennert J, Platz Batista da Silva N, Schreyer AG, Melter M, et al. Evaluation of the diagnostic accuracy of CEUS in children with benign and malignant liver lesions and portal vein anomalies. Clin Hemorheol Microcirc. 2015 Oct 5;61(2):333-45.

26. Coleman JL, Navid F, Furman WL, McCarville $\mathrm{MB}$. Safety of ultrasound contrast agents in the pe- diatric oncologic population: A single-institution experience. AJR Am Roentgenol. 2014; 202: 96670.

27. McCarvile MB, Kaste SC, Hoffer FA, Khan RB, Walton CR, Alpert BS, et al. Contrast-enhanced sonography of malignant pediatric abdominal and pelvic solid tumors: preliminary safety and feasibility date. Pediatr Radiol. 2012;42:824-33.

28. Dietrich CF, Averkiou MA, Correas JM, Lassau N, Leen E, Piscaglia F. An EFSUMB introduction into dynamic contrast-enhanced ultrasound (DCE-US) for quantification of tumor perfusion. Ultraschall in Med. 2012;33:344-51.

29. Ključevšek D, Vidmar D, Urlep D, Dežman R. Dynamic contrast-enhanced ultrasound of the bowel wall with quantitative assessment of Crohn's disease activity in childhood. Radiol Oncol. 2015; doi:10.1515/raon-2015-0042. 